\title{
PF24 Plasma
}

National Cancer Institute

\section{Source}

National Cancer Institute. PF24 Plasma. NCI Thesaurus. Code C133342.

The fluid portion of one unit of human blood intended for transfusion which has been separated from whole blood and placed at - 18 degrees C or colder within 24 hours from whole blood collection. When prepared by automated apheresis the product is stored at 1 degree to 6 degrees $C$ within 8 hours of collection and frozen at -18 degrees C or colder within 24 hours of collection. 